schmelzen. Die Lösung reagirt neutral. In Alkohol und Aether löst sich der Körper leicht. Er enthält 1 Molecul Wasser; die bei $90^{\circ}$ entwässerte Substanz bleibt bei gewöhnlicher Temperatur flüssig und erstarrt erst auf Zusatz von Wasser.

I. $0,2065 \mathrm{~g}$ gaben $0,2920 \mathrm{CO}_{2}$ und $0,0940 \mathrm{H}_{2} \mathrm{O}$.

II. $0,2450 \mathrm{~g} \cap 41,4 \mathrm{cbcm} \mathrm{N}$ bei $19^{\circ}$ und $754,5 \mathrm{~mm}$ Druck.

III. $0,3925 \mathrm{~g}$ verloren bei $90^{\circ} 0,0330 \mathrm{H}_{2} \mathrm{O}$.

\begin{tabular}{lcccc} 
& $\begin{array}{c}\text { Berechnet für } \\
\mathrm{C}_{7} \mathrm{H}_{9} \mathrm{~N}_{8} \mathrm{O}_{4}+\mathrm{H}_{8} \mathrm{O}\end{array}$ & \multicolumn{3}{c}{ Gefunden } \\
$\mathrm{C}$ & 38,71 & I. & II. & III. \\
$\mathrm{H}$ & 5,07 & 38,70 & - & - \\
$\mathrm{N}$ & 19,35 & 5,06 & - & - \\
$\mathrm{H}_{2} \mathrm{O}$ & 8,29 & - & 19,28 & - \\
& & - & - & $8,40$.
\end{tabular}

L e i pzig, den 28. März 1889.

\title{
Ueber die Oxycitraconsäure und einige ihrer Derivate ;
}

\section{von $P$. Melikoff und M. Feldmann.}

(Eingelaufen den 22. April 1889.)

Die Natur der Glycidsäuren, welche aus den einbasischen ungesättigten Säuren erhalten werden, ist in ihrer Fähigkeit, die Elemente der Halogenwasserstoffsäuren, des Wassers oder des Ammoniaks zu addiren, ausgesprochen. Um aufzuklären, wie weit die genannten Eigenschaften sich auch in denjenigen Glycidsäuren wiederfinden, welche sich von den ungesättigten zweibasischen Säuren ableiten, untersuchten wir zunächst in diesem Sinne die Citraconsäure.

Vor allem prüften wir, wie sich diese Säure gegen eine verdünnte wässerige Lösung von Unterchlorigsäurehydrat ver- 
hält. Indem wir die verdünnte wässerige Lösung der letzteren in kleinen Mengen zu der stark verdünnten Lösung der Citraconsäure zugaben, gelang es ziemlich schnell, das Ende der Reaction zu erreichen; es trat dabei weder erhebliche Wärmeentwickelung, noch bemerkenswerthe Kohlensäurebildung ein. Daraus ergiebt sich, dafs die Addition der Unterchlorigsäure zur freien Citraconsäure mit gleichem Erfolge stattfindet, wie es nach Carius*) für das Baryumsalz der Fall ist. Das Reactionsproduct wurde ferner durch Aether extrahirt und nach dem Abdestilliren des letzteren begann nach kurzer Zeit die Krystallisation der erhaltenen Chloroxysäure. Das Baryumsalz dieser Säure enthält, wie Gottlieb**) und Mor aws k i ***) für das chlorcitramalsaure Baryum angaben, 4 Mol. Krystallwasser.

1. $0,1370 \mathrm{~g}$ des Salzes gaben $0,0816 \mathrm{BaSO}_{4}$.

2. $0,1175 \mathrm{~g} n n$ n 0,0701 ,

3. $0,1867 \mathrm{~g} n$ n $0,0687 \mathrm{AgCl}$.

Berechnet füir $\mathrm{C}_{5} \mathrm{H}_{5} \mathrm{ClO}_{5} \mathrm{Ba}, 4 \mathrm{H}_{4} \mathrm{O}$

$\mathrm{Ba}$

35,17

$\mathrm{Cl}$

9,12

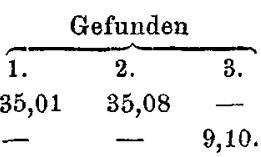

Nachdem so die Fähigkeit der freien Citraconsäure, die Elemente des Unterchlorigsäurehydrates zu addiren, nachgewiesen war, prüften wir die Darstellungsmethode der Chlorcitramalsäure von Gottliebt); dabei wurde jedesmal vor dem Chloreinleiten die nicht mehr als $2 \mathrm{pC}$. enthaltende wässerige Lösung des citraconsauren Natriums gut gekühlt, so dafs ihre Temperatur selbst bei thätigster Chlorabsorption $5^{0}$ nie überstieg. Indem wir in allem übrigen nach Gottlie b's Angaben verfuhren, erhielten wir Krystalle von seltener Gröfse,

*) Dieso Annalen 126, 204.

*) Daselbst 160, 101.

***) Journ. f. prakt. Chem. [2] I0, 68 .

†) a. a. O. S. 104 . 
deren Schmelzpunkt jedoch bei vielfacher Bestimmung immer bei $139^{\circ}$ lag und in keinem Falle bei $100^{\circ}$, wie G ottlie b angiebt. Zwei Bestimmungen des Chlors in der Säure nach Carius gaben Zahlen, welche der Zusammensetzung der Chlorcitramalsäure entsprechen :

1. $0,1936 \mathrm{~g}$ gaben $0,1554 \mathrm{AgCl}$.

2. $0,1018 \mathrm{~g}$ " 0,0815 n

\begin{tabular}{cccc} 
& Berechnet für & \multicolumn{3}{c}{ Gefunden } \\
$\mathrm{Cl} \quad$ & $\mathrm{C}_{5} \mathrm{H}_{7} \mathrm{ClO}_{5}$ & 1. & 2. \\
19,45 & & 19,54 & $19,73$.
\end{tabular}

Un aus der Chlorcitramalsäure die von Morawski beschriebene und ausführlich untersuchte Säure, die er Oxycitraconsäure nennt, zu gewinnen, benutzten wir die gewöhnliche Art der Darstellung von Glycidsäuren. Der bei Zugabe von alkoholischem Kali zu der alkoholischen Lösung der Chloroxysäure entstehende voluminöse Niederschlag wurde jedesmal durch Kochen in einigen Minuten in dünnes Pulver umgewandelt; an dieser Erscheinung erkennt man das Ende der Reaction : Abspaltung von Chlorkalium und Bildung des Kaliumsalzes der Glycidsäure. Das erhaltene Salz wurde immer, um das überschüssige Kali zu entfernen, sorgfältig mit Alkohol, dann mit Aether gewaschen; nach völligem Entfernen des letzteren wurde das Salz in Wasser gelöst, durch Schwefelsäure zerlegt und die freie Säure durch vielfaches Ausschütteln mit Aether extrahirt. Nach dem Abdestilliren des Aethers oder auch beim Abdampfen an der Luft erhielten wir die Glycidsäure in sternförmig gruppirten Krystallen, die sehr schnell verwittern und weifs werden. Mit der bei $70^{\circ}$ getrockneten Säure wurde eine Verbrennung ausgeführt.

$0,3568 \mathrm{~g}$ gaben $0,5364 \mathrm{CO}_{2}$ und $0,1400 \mathrm{H}_{2} \mathrm{O}$.

$\begin{array}{lcr} & \text { Berechnet für } & \text { Gefunden } \\ & \mathrm{C}_{5} \mathrm{H}_{6} \mathrm{O}_{5} & \\ \text { C } & 41,09 & 41,01 \\ \text { H } & 4,11 & 4,35 .\end{array}$


Die Angabe Morawski's, dafs die Säure sich bei 120 bis $130^{\circ}$ zersetze, können wir nicht bestätigen; die Säure hat allerdings keinen ausgesprochenen Schmelzpunkt, zerlegt sich jedoch erst bei $162^{\circ}$, wie auch Scherks*) angiebt. Eine ausführlichere Untersuchung der Salze dieser Säure haben wir unterlassen; zur Darstellung ihres Esters benutzten wir das Silbersalz; dieses läfst sich, wie schon Mor aw ski angiebt, nicht durch doppelte Zersetzung des Kaliumsalzes mit Silbernitrat erhalten, weil dabei Doppelsalze entstehen. Von richtiger Zusammensetzung erhielten wir das Silbersalz durch Sättigung der freien Säure mit Silbercarbonat. Es wurde neben Schwefelsäure getrocknet und mit der äquivalenten Menge Aethyljodür in absolutem Aether 5 bis 6 Stunden auf dem Wasserbade am Rückflufskühler erwärmt. Der entstandene Ester wurde vom Jodsilber abfiltrirt und fractionirt; sein Siedepunkt unter gewöhnlichem Luftdruck, wobei fast keine Verkohlung zu bemerken ist, lag immer bei 244 bis $245^{\circ}$.

1. $0,2118 \mathrm{~g}$ gaben $0,4129 \mathrm{CO}_{2}$ und $0,1321 \mathrm{H}_{2} \mathrm{O}$.

2. $0,1476 \mathrm{~g}$ n 0,2894 n 0,0935 n

\begin{tabular}{ccrr} 
& $\begin{array}{c}\text { Berechnet für } \\
\mathrm{C}\end{array}$ & $\overbrace{3} \mathrm{H}_{4} \mathrm{O}\left(\mathrm{COOC}_{2} \mathrm{H}_{5}\right)_{2}$ & \multicolumn{1}{c}{ Gefunden } \\
$\mathrm{H}$ & 53,47 & 53,16 & $\mathbf{5 3 , 4 7}$ \\
$\mathrm{H}$ & 6,93 & 6,93 & $7,03$.
\end{tabular}

Das specifische Gewicht des Esters ist auf Wasser von gleicher Temperatur bezogen bei $0^{0}=1,1376$, bei $22^{\circ}$ $=1,1167$; daraus berechnet sich sein Ausdehnungscoëfficient zu 0,0008507; mit Wasser ist der Ester nicht mischbar, in Alkohol und Aether ist er in allen Verhältnissen löslich.

Da wir die beschriebene Säure als eine glycidartige Verbindung betrachteten, setzten wir voraus, dafs sie leicht Halogenwasserstoffsäuren addire. Ueber die Addition der Chlorwasserstoffsäure giebt Morawski an, dafs sie zwar

*) Diese Annalen 228, 233. 
leicht genug stattinde, immerhin aber Erhitzen in zugeschmolzenen Röhren bis auf $110^{\circ}$ verlange. Wir haben dagegen gefunden, dafs Erhitzen vollständig zu vermeiden ist, denn die Addition verläuft schon bei gewöhnlicher Temperatur, ohne dafs Wärmeentwickelung zu bemerken wäre. Bein Uebergiefsen mit bei $0^{0}$ gesättigter Chlorwasserstoffsäure löst sich die Oxycitraconsäure ziemlich rasch und durch Aether wird aus dieser Lösung die schon entstandene Chloroxysäure extrahirt; sie krystallisirt in durchsichtigen rhombischen Tafeln, welche ihrem Aussehen nach sehr leicht von der durch Verbindung von unterchloriger Säure mit Citraconsäure entstehenden Chloroxysăure zu unterscheiden sind; ihr Schmelzpunkt fällt mit dem von Moraw ski angegebenen zusammen; liegt also wie der der Oxycitraconsäure bei $162^{n}$.

$0,3383 \mathrm{~g}$ mit Kalk geglüht gaben $0,2705 \mathrm{AgCl}$.

$\begin{array}{ccc} & \text { Berechnet für } & \text { Gefunden } \\ \text { Cl } & \mathrm{C}_{5} \mathrm{H}_{7} \mathrm{ClO} \mathrm{O}_{5} & \\ 19,45 & 19,48 .\end{array}$

Die Salze dieser Säure sind sehr unbeständig und zerlegen sich unter Bildung von Metallchloriden; es gelingt aber sie zu erhalten, indem man ihre gesättigte wässerige Lösung sogleich mit Alkohol und Aether fällt. Im Besonderen haben wir die Salze mit Baryum, Zink und Calcium aus der mit dem entsprechenden Carbonat gesättigten wässerigen Lösung der Säure auf diese Weise abgeschieden.

Calciumsalz :

$0,2362 \mathrm{~g}$ verloren bei $75^{\circ} 0,0338 \mathrm{H}_{2} \mathrm{O}$.

$0,2024 \mathrm{~g}$ entwässerten Salzes gaben $0,1245 \mathrm{CaSO}_{4}$.

$0,2277 \mathrm{~g}$ wasserenthaltenden Salzes gaben $0,1215 \mathrm{CaSO}_{4}$.

$\begin{array}{lcccc} & \begin{array}{c}\text { Berechnet für } \\ \mathrm{C}_{5} \mathrm{H}_{5} \mathrm{CaClO}_{5}+2 \mathrm{H}_{2} \mathrm{O}\end{array} & \overbrace{\mathrm{I} .}^{\text {Gefunden }} & \text { II. } \\ \mathrm{H}_{2} \mathrm{O} & 14,03 & - & \mathbf{1 4 , 3 1} \\ \mathrm{Ca} & 15,59 & 15,50 & 15,69 .\end{array}$

Dafs die Säure Bromwasserstoff zu addiren vermag, wird 
92 Melikoff u. Feldmann, über die Oxycitraconsäure

schon von $\mathrm{Scherks}$ in seiner oben angeführten Arbeit *) erörtert. Indem wir nach Scherks Angaben verfuhren, lösten wir die Glycidsäure in bei $0^{\natural}$ gesättigter Bromwasserstoffsäure und erhielten in einigen Stunden kleine durchsichtige Krystalle, deren Schmelzpunkt übereinstimmend mit Scherks bei $156^{\circ}$ gefunden wurde.

$0,3028 \mathrm{~g}$ mit Kalk geglüht gaben $0,2499 \mathrm{AgBr}$.

$$
\begin{array}{ccc} 
& \text { Berechnet für } & \text { Gefunden } \\
\mathrm{C} & \mathrm{C}_{5} \mathrm{H}_{7} \mathrm{BrO}_{5} & \\
\mathrm{Br} & 35,24 & 35,11 .
\end{array}
$$

Um die Constitution der Oxycitraconsäure entscheidend festzustellen und zu beweisen, dafs sie ein der Glycidsäure analoges Anhydrid ist, war es nothwendig zu untersuchen, ob sie die Fähigkeit besitze, die Elemente des Ammoniaks zu addiren, da diese Eigenschaft für die einbasischen Glycidsäuren besonders charakteristisch erscheint. Die Oxycitraconsäure wurde dazu in kleinen Mengen wässerigen Ammoniaks gelöst und die Lösung mit Ammoniak bei $0^{0}$ gesättigt. Um die mögliche Bildung von Citraweinsäure zu vermeiden, setzten wir der erhaltenen Lösung eine bei $0^{0}$ gesättigte Lösung von Ammoniak in Alkohol zu, bis die Flüssigkeit sich zu trüben begann. Die Mischung wurde in einem zugeschmolzenen Rohre 5 Stunden lang auf $100^{\circ}$ erhitzt. Nach dem Oeffnen des Rohres und Abdampfen seines Inhaltes schieden sich lange nadelförmige Krystalle des Ammoniumsalzes der neu entstandenen Amidosäure aus. Die Krystalle wurden abgeprefst, in Wasser gelöst und mit frisch gefälltem Bleioxyd so lange erwärmt, bis alles Ammoniak ausgetrieben war; die ganze Masse wurde dann durch Schwefelwasserstoff zerlegt, das Schwefelblei abfiltrirt und das Filtrat auf dem Wasserbade eingedampft. Aus der gesättigten Lösung schied sich die Amidosäure in grofsen durchsichtigen Krystallen aus.

*) a. a. O. Seite 240 . 
Amidocitramalsäure, $\mathrm{C}_{3} \mathrm{H}_{4}\left(\mathrm{NH}_{2}\right) \mathrm{OH}(\mathrm{COOH})_{2}$, krystallisirt in durchsichtigen abgekürzten Prismen, die dem monoklinen System anzugehören scheinen; einzelne Krystalle erreichen die Länge von $5 \mathrm{~mm}$ bei $1 \mathrm{~mm}$ Dicke. Die Amidosäure hat einen scharf sauren Geschmack und färbt Lackmus roth, womit sie sich von den einbasischen Amidoglycidsäuren unterscheidet; aufserdem besitzt sie die Eigenschaft Carbonate unter Entwickelung von Kohlensäure zu zerlegen; sie ist in Wasser löslich : 100 Gewichtstheile Wasser von $18^{\circ}$ lösen 31 Theile Amidosäure; in heifsem Alkohol ist sie fast unlöslich.

$0,1612 \mathrm{~g}$ Substanz mit $\mathrm{CuO}$ verbrannt gaben $11,3 \mathrm{cbcm}$ feuchten Stickstoff bei $761 \mathrm{~mm}$ Druck und $15^{\circ}$.

Berechnet

$\mathrm{N}$ 8,48 Gefunden 8,22 .

Das Calciumsalz, durch Sättigung der wässerigen Lösung der Säure mit Calciumcarbonat dargestellt, verblieb nach dem Abdampfen auf dem Wasserbade als amorpher Niederschlag. Einige Calciumbestimmungen zeigten, dafs das Salz die $\mathrm{Zu}-$ sammensetzung hat :

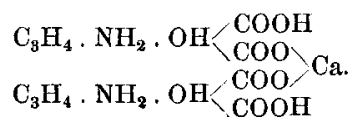

$0,1554 \mathrm{~g}$ bei 100 getrocknetes Calciumsalz gaben $0,0580 \mathrm{CaSO}_{4}$.

Berechnet

$\mathrm{Ca}$ 10,99
Gefunden 10,97 .

Die Säure erscheint sonach einbasisch. Wenn wir uns erinnern, dafs die einbasischen Amidooxysäuren die Carbonate gar nicht zu zersetzen vermögen, so müssen wir annehmen, dafs die genannte Eigenschaft in der Amidocitramalsäure durch die Anwesenheit des zweiten Carboxyls bedingt ist.

Das Baryumsalz, ebenso wie das Calciumsalz erhalten, ist auch amorph; die Analyse zeigte wiederum, dafs die Säure einbasisch ist.

$0,2524 \mathrm{~g}$ gaben $0,1287 \mathrm{BaSO}_{4}$.

Berechnet

Ba
Gefunden 29,97 . 
94 Melikoff u. Feldmann, über die Oxycitraconsäure

Das Kupfersalz wird durch Sättigung der wässerigen Lösung der Säure mit Kupferhydroxyd erhalten und besitzt in wässeriger Lösung die charakteristische dunkelblaue Farbe; im trocknen Zustande stellt es eine amorphe blaue Masse vor.

Obgleich die Amidocitramalsäure sich wie eine ächte Säure verhält, so kann sie sich doch auch mit Halogenwasserstoffsäuren verbinden. Beim Auflösen in Chlorwasserstoffsäure und Abdampfen an der Luft scheidet sich die Verbindung $\mathrm{C}_{3} \mathrm{H}_{4}\left(\mathrm{NH}_{2}\right) \mathrm{OH}(\mathrm{COOH})_{2}+\mathrm{HCl}$ in schiefen durchsichtigen Prismen aus, die in Wasser und heifsem Alkohol leicht löslich sind; beim Erwärmen auf $100^{\circ}$ schmelzen die Krystalle, indem sie sich in eine glasartige Masse umwandeln und theilweise Chlorwasserstoff verlieren.

Auf Grund aller bewiesenen Thatsachen über die Addition der Elemente der Halogenwasserstoffsäuren und des Ammoniaks ist die Oxycitraconsäure Morawski's eine Glycidsäure. Die Angehörigkeit dieser Säure zu den Glycidverbindungen wird noch dadurch bestätigt, dafs die Säure mit Hydroxylamin sich nicht verbindet und ihr Ester mit Kaliumäthylat nicht reagirt.

Die Bildung der Glycidsäure aus der Citraconsäure läfst sich durch folgende Gleichungen erklären :

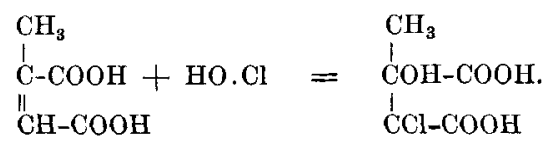

Die dabei entstehende Chlorcitramalsäure muf's unvermeidlich solche Constitution besitzen, da nur diese Formel die Entstehung der Chlorcitraconsäure bei der trocknen Destillation der Chlorcitramalsäure zu erklären im Stande ist*). Die Bildung der Glycidsäure aus dieser Chloroxysäure geschieht also nach folgender Gleichung :

*) Gottlieb, Journ. f. prakt. Chem. [2] 8, 73 . 


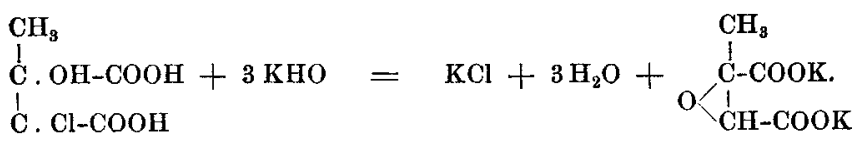

Die Entstehung der isomeren Chlorcitramalsäure bei der Addition von $\mathrm{HCl}$ zu der Glycidsäure hat ihren Ausdruck in der Gleichung :

$$
\begin{aligned}
& \mathrm{CH}_{3} \\
& 1 \\
& \mathrm{C}-\mathrm{COOH}+\mathrm{HCl} \\
& 1 \\
& \mathrm{CH}-\mathrm{COOH}
\end{aligned}=\begin{gathered}
\mathrm{CH}_{3} \\
1 \\
\mathrm{Cl}-\mathrm{C}-\mathrm{COOH} \\
\quad \text { HO-CH-COOH }
\end{gathered} .
$$

Da endlich die Frage über die Vertheilung der Elemente des Ammoniaks offen steht, so kann die Constitution der Amidocitramalsäure durch eine der folgenden zwei Formeln ausgedrückt werden :

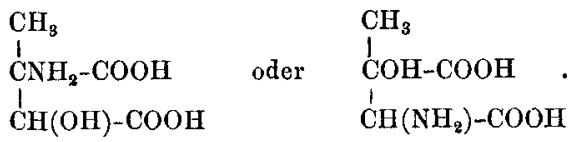

O de s s a, Universität.

Ein Versuch zur Bestimmung der Dampfdichte des Antimonpentachlorids unter vermindertem Druck;

von Richard Anschiltz und Norman P. Evans.

(Hierzu Tafel I.)

[Mittheilung aus dem chemischen Institut der Universität Bonn.]

In der Einleitung einer vor zwei Jahren in diesen Annalen *) veröffentlichten Abhandlung : „Beiträge zur Kenntnifs des Antimonpentachlorids" haben wir unsere Mifserfolge bei

*) Diese Annalen $\mathbf{2 3 9}, 285$. 